\title{
Hombres académicos y distanciamiento social: reflexiones en torno a lo doméstico, el trabajo y la masculinidad
}

\section{Male Academics and Social Distancing: \\ Reflections on the Domestic, Work and Masculinity}

Alí Siles

Centro de Investigaciones y Estudios de Género, Universidad Nacional Autónoma de México, Ciudad de México, México.

email:ali_siles@cieg.unam.mx, [0 https://orcid.org/oooo-0oo2-3878-8070

\section{Recibido: 20 de}

noviembre 2020

Aceptado: o1 de junio de 2021

Publicado: 12 de julio de 2021

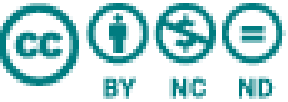

Esta obra está protegida bajo una Licencia Creative Commons Atribución-NoComercialSinDerivadas 4.0 Internacional (CC BY-NC-ND 4.0)

\section{Resumen}

Este artículo discute algunos de los hallazgos respecto de la experiencia de varones en la labor académica durante el confinamiento (derivado de las medidas impuestas a raíz de la aparición de la COVID-19). Se analizan las narrativas de veinte hombres de distintas áreas de conocimiento y en diversas posiciones académicas en la Universidad Nacional Autónoma de México, UNAM. Sus relatos, arrojan luz acerca de cómo se producen y reproducen masculinidades “tradicionales" en la labor académica, adecuada a las medidas de distanciamiento social, y sobre la estructura de relaciones de género que enmarca la experiencia cotidiana de estos varones. El argumento central es que el confinamiento ilumina la existencia de modelos "tradicionales" de masculinidad entre los académicos que reproducen el espacio doméstico

CÓMO CITAR: Siles, Alí. (2021). Hombres académicos y distanciamiento social: reflexiones en torno a lo doméstico, el trabajo y la masculinidad. Revista Interdisciplinaria de Estudios de Género de El Colegio de México, 7, e724. doi: http://dx.doi.org/10.24201/reg.v7i1.724 
como feminizado y que, simultáneamente, pueden acomodar reconfiguraciones de lo doméstico (por medio de prácticas asociadas a las llamadas "nuevas masculinidades") y propiciar la reflexividad entre los varones en torno a sus prácticas, identidades y relaciones de género.

Palabras clave: Hombres; masculinidad; trabajo doméstico; personal académico; distanciamiento social, confinamiento.

\begin{abstract}
This article discusses some findings of research on male academics and their experience of working under the enforced social distancing measures put in place after the COVID-19 outbreak. By analyzing the narratives of twenty male academics from the Universidad Nacional Autónoma de México, UNAM working in different fields and posts within the university, this study sheds light on how "traditional" masculinities are produced and reproduced in academic work in line with social distancing measures, and on the gender relations structure that frames their daily lives as part of the university's academic staff. The core argument is that confinement reveals the existence of "traditional" models of masculinity among academics who reproduce the domestic space as feminized and that can, simultaneously accommodate a reshaping of the domestic (through practices resemblant of so-called "new masculinities") and promote reflexivity among men about their gender practices, identities, and relations.
\end{abstract}

Keywords: men; masculinity; housework; academic staff; social distancing; confinement.

\title{
Introducción
}

Las medidas de distanciamiento social implementadas tras la irrupción y propagación del virus SARS-COV-2 y la enfermedad que provoca (denominada COVID-19) han impactado el espectro de actividades humanas casi en su totalidad. De manera sobresaliente, ha derivado en el confinamiento de amplios sectores de la población ${ }^{1}$ en sus lugares de residencia por

\footnotetext{
${ }^{1}$ La excepción más notable en este sentido es, sin duda, la del personal llamado "esencial" entre quienes destacan los de las áreas de salud, limpieza, y otros servicios para la producción, distribución y proveeduría de bienes y servicios considerados como de primera necesidad.
} 
periodos inusualmente prolongados. Al reducir la movilidad de las personas y extender el tiempo que pasan en sus hogares - a veces por varios días o semanas consecutivas - el confinamiento ha dado lugar a reajustes en las dinámicas que se desarrollan en el espacio doméstico, particularmente, en términos de las relaciones e interacciones con base en el género.

Muchas de las personas, cuyos centros de trabajo han sido cerrados, han tenido que adecuar sus labores para realizarlas desde sus lugares de residencia a través de medios electrónicos. Ello ha implicado la adaptación de los espacios y una afectación en los tiempos y movimientos habituales en ellos. Éste ha sido el caso de trabajadoras y trabajadores en distintas posiciones y dimensiones de la labor académica, quienes han tenido que adaptar su trabajo de docencia, investigación y administración a estas nuevas condiciones. Ello implica una interacción particular entre, por un lado, los arreglos relacionales y las prácticas e identidades de género que cotidianamente configuran los espacios laborales académicos y operan en ellos y, por otro, aquellas que organizan los espacios domésticos y las actividades ahí realizadas.

El presente artículo explora algunos de los efectos de las medidas de distanciamiento social y confinamiento en la vivencia de trabajadores académicos de diversas áreas de conocimiento y con distintos nombramientos en la Universidad Nacional Autónoma de México (UNAM) ${ }^{2}$. Se trata de observar cómo esta circunstancia excepcional pone de manifiesto elementos de la producción y reproducción de masculinidades.

Luego de ofrecer algunas consideraciones teóricas respecto a los vínculos entre masculinidades, trabajo académico y espacio doméstico, hago una breve descripción de la metodología implicada en la investigación. Posteriormente, analizo en tres secciones la vivencia de los varones en el confinamiento y la reflexividad que ésta ha generado en ellos. La primera parte tiene que ver con el trabajo académico como una actividad altamente masculinizada, que descansa de manera importante sobre la (re)producción de lo doméstico como mayormente feminizado. Los privilegios (masculinos) de la labor académica se distribuyen de manera diferenciada entre estos varones según su estatus contractual,

\footnotetext{
${ }^{2}$ Los datos y el análisis presentados en este texto forman parte de un proyecto de investigación - más amplioen curso en el Centro de Investigaciones y Estudios de Género (CIEG) de la UNAM, titulado Masculinidades universitarias frente al cambio en las relaciones de género: alcances y desafios.
} 
trayectoria académica, nivel socioeconómico y otros elementos estructurales. En este sentido, resalta el papel de la división sexual del trabajo, ejemplificada claramente por la contratación de trabajadoras que asisten en la limpieza y mantenimiento de los hogares de varios de estos académicos.

La segunda sección discute los modelos específicos de masculinidad que aparecen en las narrativas de los participantes. Estos modelos incluyen patrones de arreglos y prácticas de masculinidad convencionales que responden, en varios sentidos, a la noción de masculinidad hegemónica (Connell, 1987; 2015; Messerschmidt, 2018), así como aspectos de las llamadas "nuevas masculinidades". Estas prácticas de masculinidad que pueden ser caracterizadas como "nuevas" corresponden a una serie de condiciones materiales que el trabajo académico concede a algunos de los participantes, a su experiencia de vida (su pertenencia a grupos etarios determinados, su estatus socioeconómico de origen y actual, etcétera) y que, de manera sobresaliente, cobran particular vigencia como respuesta a las condiciones impuestas por el confinamiento.

La tercera y última sección explora momentos y espacios de reflexividad que surgen entre los participantes en el contexto del confinamiento. En este sentido, destacan ejemplos de varones que han iniciado un proceso de reflexión que incluye elementos de una perspectiva de género de maneras tangenciales, así como de académicos que han podido profundizar en un proceso de resignificación de sus posiciones y relaciones de género, iniciado previamente al advenimiento de lo que los participantes llaman "la pandemia".

\section{Masculinidades, trabajo académico y espacio doméstico: consideraciones teóricas}

El género, entendido como un complejo entramado de relaciones, prácticas y significados sociales y culturales que regulan y estructuran las interacciones entre cuerpos masculinizados y feminizados, ha asignado a lo doméstico un carácter natural y primordialmente "femenino". De manera simultánea, los mismos procesos relacionales y de significación han asociado históricamente el espacio extradoméstico con "lo masculino". Esta disposición género-espacial ha descansado, en gran medida, sobre una diferenciación de los cuerpos con base en sus capacidades reproductivas (Connell y Pearse, 2015) y en las 
funciones que se les asignan en ese sentido ${ }^{3}$. Así, esta configuración económico-social, con la división sexual del trabajo como núcleo, ha definido dos grandes espacios en los que se localizan las nociones de lo doméstico y lo público como su contraparte: "el hogar" y "el trabajo".

Los estudios críticos sobre varones han establecido con claridad la construcción de la masculinidad como "manifiesta espacialmente en la separación entre el hogar y el trabajo" (Smith y Winchester, 1998, p. 328, traducción propia). Más allá de las múltiples formas en las que el orden de género imperante en las sociedades occidentales (y occidentalizadas) (Connell y Pearse, 2015) excluye a las mujeres de diversos espacios de trabajo, la investigación sobre masculinidades ha aportado profundidad al entendimiento de cómo el trabajo extradoméstico, remunerado o productivo, ha estado y se mantiene masculinizado, y las diversas formas en que éste ha moldeado prácticas e identidades de género (Figueroa, 2014; Olavarría, 2001; 2017).

Por otro lado, la literatura sobre masculinidades también ha dado cuenta del papel de lo doméstico en la configuración de identidades masculinas, especialmente por medio de los procesos que tienen lugar al interior de las familias (Olavarría, 2017), y de cómo el hogar puede ser visto como un sitio profundamente masculino. Como lo plantea Madhura Lohokare (2020), "las masculinidades son esencialmente relacionales: construidas vía interacciones complejas entre hombres, mujeres, hijas e hijos en el contexto espacial profundamente 'privado' demarcado como hogar/familia” (p. 293, traducción propia).

Aun cuando los espacios de trabajo extradoméstico son todavía un sitio de práctica e identidad masculina hegemónica muy importante, estas identidades tienen necesariamente un correlato con las interacciones, prácticas e identidades de género producidas en el ámbito de lo doméstico. La investigación sobre masculinidades en América Latina (Figueroa, 2014) y en otras latitudes (Smith y Winchester, 1998) ha documentado cómo los hogares pueden ser sitios propicios para la negociación de masculinidades, especialmente en torno a la contribución de los varones en las labores domésticas, y de cómo las condiciones

\footnotetext{
${ }^{3}$ Esto es, cuerpos “femeninos": gestación y alumbramiento, cuidado, crianza; cuerpos "masculinos": provisión, protección. Ello confiere especial importancia a las categorías de "masculinidad" y "feminidad" como formativas e informativas de la distinción entre cuerpos y de lo que dicha distinción implica en términos de prácticas corporales sociales y culturales cotidianas (Connell, 1983).
} 
estructurales cambiantes ligadas a reacomodos en el sistema de producción global y el mercado laboral impactan dicha negociación.

$\mathrm{Si}$ bien la configuración género-espacial que construye lo doméstico como predominantemente "femenino" y los espacios laborales como mayormente "masculinos" ha sido objeto de críticas, resistencia y contestación en México (con impactos diversos), en las primeras décadas del siglo XXI, aún exhibe importantes continuidades en la experiencia vivida de mujeres y hombres en diversos ámbitos, momentos y espacios de la vida colectiva. En este sentido, la literatura (Buquet, 2016; Buquet, Cooper, Mingo y Moreno, 2013; Cerva, 2018; Ríos, Mandiola y Varas 2017; Olavarría, 2017) da cuenta de cómo, en gran medida, dicha configuración da forma a la labor y experiencia de mujeres y varones dedicados al trabajo académico.

Tal como lo señalan Buquet et al. (2013), el orden de género ha jugado un papel fundamental en dar forma a lo que las autoras llaman "la cultura institucional universitaria" (p. 284). De manera muy atinada, también advierten que "la situación de privilegio de que disfrutan los varones por el solo hecho de pertenecer al sexo masculino" (p. 284) es un factor fundamental para considerar en la vivencia de las y los académicos, y que la condición desigual global entre éstos "es interpretada desde una posición de privilegio no percibida, como inevitable, justa o necesaria" (p. 284).

Por otro lado, más allá de las condiciones estructurales (entre ellas la conformación géneroespacial femenino/espacio doméstico, masculino/espacio público) que han perpetuado esa desigualdad, es oportuno prestar atención a la configuración y reconfiguración de prácticas e identidades de género que tienen lugar a través de esas estructuras y que, a su vez, tienen la potencialidad de reproducirlas, reconstituirlas, resignificarlas o subvertirlas. En este sentido, mi propuesta es explorar esas potencialidades en términos de identidades masculinas, por parte de los varones académicos, que tendrían lugar en los espacios domésticos que construyen y habitan.

Las masculinidades pueden ser entendidas como esquemas que definen ámbitos de poder, organizan el acceso a recursos y —notablemente- segregan los espacios sociales (Amuchástegui y Szasz, 2007), entre ellos, el del trabajo académico. Dichos espacios son organizados por los cuerpos que los ocupan y éstos, a su vez, se constituyen por medio de 
los primeros, es decir cuerpo y espacio son co-constituyentes (Gorman-Murray, 2013). Así pues, ni la domesticidad ni la masculinidad son fijas y permanentes, sino que existe entre ellas una relación de mutua y constante co-constitución (Gorman-Murray, 2008). De este modo, es posible pensar las masculinidades de los varones dedicados al trabajo académico en continua (re)formación, tanto por su relación con el espacio laboral académico, como por sus interacciones y vivencias con, de, y desde lo doméstico, que están sujetas a procesos de configuración y reconfiguración (más o menos) constantes.

Estos procesos de (re)configuración y co-constitución incluirían —aunque no estarían limitados - instancias de contestación, resistencia o adecuación respecto al curso y las dinámicas de la interacción de los varones académicos con las personas con quienes cohabitan o comparten de alguna manera el espacio doméstico. Tales instancias se ligan a momentos, situaciones o eventos específicos. En este sentido, el actual contexto de confinamiento ofrece una importante oportunidad para indagar respecto a la experiencia y (re)producción de masculinidades entre varones académicos, los procesos mediante los cuales sus prácticas e identidad masculinas pueden ser negociadas y (re)configuradas, y los espacios en los que esos procesos transcurren.

\section{Propuesta metodológica}

Las condiciones de movilidad e interacción resultantes de las medidas implantadas por la irrupción de la COVID-19 han orillado a investigadoras e investigadores a realizar una gran parte de su labor a través de medios y estrategias digitales. Así, se ha generado un corpus de aproximaciones teóricas y empíricas acerca de los efectos que las medidas de distanciamiento social han provocado, observados desde diversas disciplinas científicas y humanísticas e incorporando la perspectiva de género. De entre esos trabajos, una gran mayoría han prestado atención a las formas en que las desigualdades de género previamente existentes se han visto agudizadas a raíz de la declaratoria de pandemia (véase, por ejemplo, Castellanos-Torres, Mateos y Chilet-Rosell 2020; del Río y García, 2020; Ruíz, 2020, Wenham, Smith y Morgan, 2020) o al urgente fenómeno de la exacerbación de la violencia contra mujeres, niñas y niños durante los periodos más intensos de confinamiento (véase, por ejemplo, López-Hernández y Rubio-Amores, 2020; Martínez-Restrepo, Tafur, Osio y Cortés, 2020; Ruiz-Pérez y Pastor-Moreno, 2020). Estos esfuerzos han utilizado 
herramientas electrónicas para la generación e interpretación de datos, principalmente en términos cuantitativos. Ello ha permitido establecer que, en efecto, el confinamiento ha impactado en las relaciones e interacciones de género, y hace observable varios de esos impactos.

Por otro lado, indagar en torno a cómo experimentan el confinamiento las personas, así como los procesos que influyen en la configuración de prácticas e identidades de género, en relación con los espacios en los que constantemente se generan y cobran forma, implica cuestionarse procesos mediante los cuales se interpreta, experimenta, produce o constituye el mundo social. Para ello, una perspectiva metodológica cualitativa resulta particularmente apropiada (Mason, 2002).

El interés por estos procesos está informado por una epistemología fenomenológica que implica pensar que la vida social transcurre en realidades múltiples y complejas que se influyen e interactúan entre sí. Siguiendo a Schutz (1967 [1932]) se parte de la premisa epistemológica de que, en términos de la vida cotidiana, las personas asumimos una "actitud natural" frente a la realidad. Dicha actitud nos permite suponer un mundo social externo a nosotras, y que las demás personas lo perciben de manera similar. Una epistemología fenomenológica implica observar las acciones realizadas por las personas de manera cotidiana - asumidas por ellas como naturales - desde una perspectiva que cuestione dicha naturalidad para buscar comprender su significado (Schutz, 1967 [1932]).

La postura epistemológica de la fenomenología propuesta por Schutz ha constituido "una base protosociológica para la metodología de las ciencias sociales" (Eberle, 2014, p. 184, traducción propia), que se ha solidificado especialmente en métodos y técnicas de investigación sociológica cualitativa. Así, tanto la concepción de esta investigación, como la generación y el análisis de los datos, se realiza con un enfoque metodológico cualitativo.

Los datos para la presente investigación fueron generados por medio de entrevistas en profundidad. Este formato de entrevista es empleado en virtud de su flexibilidad para abordar el tema a investigar de maneras diversas, adecuadas a las circunstancias específicas de los participantes, así como de la posibilidad que ofrece para alcanzar niveles de profundidad y complejidad en los datos que difícilmente pueden obtenerse a través de otros métodos, especialmente aquellos basados en encuestas (Byrne, 2016). Dadas las condiciones del 
campo de estudio ${ }^{4}$, así como las disrupciones en la vida universitaria derivadas de la contingencia sanitaria, se recurrió a una combinación de muestreo de conveniencia y por la técnica de bola de nieve ${ }^{5}$. Se entrevistó a veinte varones trabajadores académicos adscritos a diez diferentes facultades, institutos y escuelas de la UNAM, con nombramientos académicos diversos. Las entrevistas fueron realizadas entre los meses de junio y septiembre de 2020, a través de video llamadas, por la plataforma Zoom, que fueron grabadas y transcritas.

Posteriormente, los datos así generados fueron analizados con apego a un método de análisis narrativo (Esin, Fathi y Squire, 2014). La intención de este método es observar la diversidad de niveles involucrados en las narrativas de los entrevistados y buscar comprenderlas, en ese sentido, más que considerarlas como entidades unificadas, naturales y coherentes (Andrews, Day Sclater, Squire y Tamboukou, 2004). La propia experiencia subjetiva del investigador asiste en mantener el proceso analítico controlado y sistematizado (Eberle, 2014). En el caso del presente trabajo, lo anterior es aún más evidente si se considera que la experiencia de investigación de quien la conduce transcurre en circunstancias muy similares a las que experimentan los participantes ${ }^{6}$.

\footnotetext{
${ }^{4}$ Más de cuarenta mil personas realizan labores académicas remuneradas en diversas instancias y espacios de la UNAM, distribuidas a lo largo y ancho de la estructura universitaria compuesta por quince facultades, cinco unidades multidisciplinarias y nueve escuelas nacionales de educación superior, así como treinta y cuatro institutos, catorce centros y doce programas universitarios de investigación. De entre esas personas, $55 \%$ son varones: alrededor de dos mil trabajadores cuentan con nombramientos como investigadores, poco más de dos mil como técnicos académicos, y cerca de tres mil son profesores de carrera. Asimismo, aproximadamente quince mil profesores laboran bajo la figura de profesores de asignatura. Estos nombramientos implican responsabilidades académicas, condiciones de trabajo, situaciones contractuales e ingresos económicos muy diversos entre sí, abonando a la complejidad y heterogeneidad de la población académica, por lo que resulta inviable pretender una representatividad estadística en una muestra para un esfuerzo de investigación cualitativa como el presente.

${ }^{5}$ Cabe señalar que el proyecto de investigación en el que se enmarca el presente artículo diseñado previamente a la irrupción de la COVID-19, tuvo que ser acotado y redefinido de manera importante. Originalmente, se contemplaba la realización de trabajo etnográfico en diversas facultades e institutos de la UNAM. Una vez suspendidas las actividades en las instalaciones de la universidad, la posibilidad de contactar a un número amplio de participantes, dialogar con ellos de manera informal y recurrente, así como observar la configuración de los espacios físicos que habitualmente habitan y sus interacciones en ellos se vio cancelada, obligando a redefinir la estrategia para la generación de datos.

${ }^{6}$ Esto es, quien investiga lo hace también en condiciones de confinamiento y de una labor académica basada en herramientas electrónicas y digitales. Estas circunstancias generan, por sí mismas, una realidad social compleja que presenta desafíos particulares para la generación de datos, la observación de espacios, etcétera.
} 


\section{La labor académica como privilegio masculino y diferenciado}

"Yo creo que estoy muy irascible, y con la mecha muy corta; a pesar de que yo sé que estoy en una situación privilegiada". Ésta fue la primera reacción de $\operatorname{David}^{7}$, un profesor e investigador exitoso y reconocido en su área de conocimiento, a la pregunta de cómo se sentía luego de pasar varias semanas encerrado en casa. Permanecer aislado, sin poder ver a su única hija o a sus amistades más allegadas, y tratar de conducir a distancia los cursos a su cargo estaba lejos de ser una situación ideal. Y, sin embargo, como él mismo lo apunta, la suya es una situación de privilegio en muchos sentidos. A sus 67 años, ha sido profesor titular en la UNAM por más de 35, lo que le asegura un ingreso estable y suficiente. Vive solo, en una vivienda amplia y dispuesta de manera agradable, lo que por años le ha permitido me dice- distribuir sus tiempos y espacios de una manera "muy placentera" entre su trabajo, su pareja, su familia y sus amistades, hasta antes del confinamiento.

Las circunstancias impuestas por el distanciamiento social han puesto en perspectiva varios de estos privilegios para algunos académicos, incluyendo a David. Confinado en casa, no puede disfrutar de la atmósfera que habitualmente experimenta en los salones de la facultad donde imparte clases (una actividad que le resulta particularmente placentera), ni entablar conversaciones intelectual y anímicamente estimulantes con sus colegas en los pasillos u otros espacios de la universidad. Más aún, el confinamiento lo ha confrontado con un elemento que, aunque clave en su cotidianidad y, en esa medida, muy relevante para la realización de su labor académica, nunca había sido una preocupación para él:

Para mí el cambio fuerte fue la pandemia. Porque ahí empecé a hacer actividades que jamás había hecho, esa es la pura verdad: lavar los trastes, lavar la ropa, colgar la ropa; actividades que, la verdad, yo no hacía. Tampoco mi mujer [cuando estaba casado y vivía con su cónyuge]... Pero por la pandemia, la señora que venía aquí, quedamos de que no viniera; aunque mantuviera su lazo laboral que ella se quedara en su casa.

Si bien es cierto que la situación global de David dista de ser la norma entre varones académicos de la UNAM, tampoco se trata de algo excepcional. La posibilidad de que alguien

\footnotetext{
${ }^{7}$ En todos los casos, se utilizan pseudónimos en sustitución de los nombres reales de los participantes.
} 
— generalmente una mujer - "ayude" a realizar el trabajo doméstico, en su totalidad o parcialmente y de manera remunerada o no, es un elemento común en la experiencia doméstica de la mayoría de los varones académicos entrevistados.

La feminización del trabajo reproductivo, esto es, las actividades destinadas al cuidado de los hogares y familias (Carrasquer, Torns, Tejero y Romero, 1998) ha sido un tema central para la investigación de género en las últimas dos décadas. Se ha generado un importante cuerpo de literatura desde una perspectiva económica y social (véase, por ejemplo, Cruz, Noriega y Garduño, 2003; Pedrero, 2004; Espino, 2010), y significativos esfuerzos de cuantificación y valorización del trabajo reproductivo con un alto nivel de sofisticación teórico-metodológica en la región latinoamericana (véase, por ejemplo, DANE, 2020; INEGI, 2019; Inmujeres, 2017).

Así, existen bases sólidas para pensar al trabajo doméstico realizado por trabajadoras del $\operatorname{hogar}^{8}$ como una labor con un valor estratégico para la actividad académica de estos varones, de sus parejas (algunas de ellas también académicas), así como de varios integrantes de la academia. De manera notable, la feminización del trabajo reproductivo, estrechamente vinculado al espacio doméstico en muchos sentidos, aparece naturalizada en las narrativas de varios de los participantes. En ellas, lo doméstico figura como un espacio funcional, ampliamente resuelto en términos físicos, que se vuelve problemático solamente a la luz de la experiencia sui generis de vivir en confinamiento. "La pandemia" se revela como un evento disruptivo que hace evidente dicha feminización y obliga a los participantes a reacomodar sus prácticas de género habituales a la nueva realidad. Para algunos de ellos, esto implica hacerse cargo de labores reproductivas por primera vez en sus vidas. Ello presenta, además, una posibilidad para la reflexividad en torno a ella, que algunos varones abrazan, pero que en varios otros casos pasa desapercibida.

Otro ejemplo es lo señalado por Raúl al considerar cómo la ausencia de la mujer que trabaja en su casa ha impactado en prácticas habituales de su domesticidad:

\footnotetext{
${ }^{8}$ Se utiliza el término en sustitución de "trabajadora doméstica" (utilizado por algunos participantes) porque algunas trabajadoras lo rechazan por las connotaciones de sumisión y domesticación históricamente asociadas con él (Rojas y Contreras, 2018).
} 
(D)e repente descubrimos [su esposa y él] que nos toma un día entero limpiar la casa y es nuestro fin de semana, que en realidad tendría que ser el tiempo cuando realmente podemos estar juntos todo el día. Y pues, nos toca ahora tomar un día y estar juntos, pero estar limpiando, estar trabajando (Raúl, 46, investigador titular).

La disrupción en la dinámica de mantenimiento físico del hogar se refleja tanto en la producción académica de Raúl ${ }^{9}$, como en el tiempo de reproducción. Éste incluye el esparcimiento, el consumo cultural y la convivencia en pareja como elementos importantes, que aparecen altamente valorados por él y por la mayoría de los entrevistados. Los relatos de varios de ellos apuntan a la existencia de una masculinidad académica de clase media, que depende del trabajo doméstico remunerado para ser realizada en términos laborales, sociales, de esparcimiento, de consumo cultural, etcétera.

Lo anterior es particularmente claro en las narrativas de varones cuyas descripciones de sus familias de origen corresponden a una configuración familiar de clase media. Asimismo, varones procedentes de familias de clase trabajadora reportaron la adopción de la práctica de emplear a una mujer para "ayudar" con el trabajo doméstico, liberando así recursos temporales para la realización de las labores académicas, de cuidado de hijas e hijos, sobre todo en términos lúdicos, y para el consumo cultural y el esparcimiento en pareja.

Por otro lado, el hecho de que sean mayoritariamente mujeres (trabajadoras del hogar, esposas/parejas, madres/suegras, etcétera) quienes se ocupen de las labores de reproducción en los entornos domésticos de varios de los participantes es evidencia de la persistencia de la configuración género-espacial feminidad/espacio doméstico masculinidad/espacio público (en este caso, académico) del orden de género (Connell, 2015) prevalente en el contexto mexicano ${ }^{10}$. Una de las principales instituciones que participan en la configuración de dicho orden de género es, sin duda, el mercado laboral. En ese sentido, las posiciones y condiciones de privilegio que otorga a los varones la labor académica están también

\footnotetext{
${ }^{9}$ Enfatiza que resolver un problema matemático o desentrañar un párrafo de un artículo puede llevarle también un día entero de trabajo

${ }^{10}$ Esto resuena con lo planteado por Ana Buquet (2016) respecto a los efectos del orden de género en el ámbito de la investigación y la educación superior, al subrayar cómo las desigualdades en ese rubro son en gran medida resultado de las interacciones de dichas esferas con elementos del "orden cultural que permean a las sociedades en su conjunto; de las identidades que confluyen a través de procesos intersubjetivos para recrear los imaginarios colectivos; y de las estructuras de otras instituciones" (Buquet, 2016, p. 30).
} 
configuradas por el entramado relacional de la masculinidad hegemónica (Connell, 2015; Messerschmidt, 2018,) que jerarquiza la producción en el trabajo académico.

Así lo sugiere el relato de Antonio (29), quien es profesor de asignatura en la UNAM y recientemente accedió a una posición de profesor investigador de tiempo completo en otra institución de educación superior:

(N)o sé cómo la estén pasando mis colegas que son exclusivamente profesores de asignatura en este contexto de pandemia. O sea, me pongo en su lugar, si yo sólo tuviera este punto de vista de asignatura, sería complejo. No sé si eso te vicie o pueda ser como una visión dual, en esa comparación de saber las vicisitudes de la precarización del trabajo académico de profesor de asignatura y las comodidades que te puede dar la certeza de una estadía laboral en un tiempo completo [...] Pero, en desarrollo profesional y en cuestión, insisto, de percepción económica son cosas totalmente diferentes.

Antonio resalta que la seguridad y estabilidad en términos materiales que proporciona el trabajo académico, como factores que impactan de manera positiva la experiencia del confinamiento, están supeditadas a las condiciones de incorporación al trabajo y a su desarrollo. Ésta ha sido una situación sorteada de manera mayormente exitosa por los varones entrevistados que se encuentran adscritos a la universidad como profesores de asignatura $^{11}$. De manera notable, el espacio doméstico en esos casos ha funcionado como suplementario en la provisión material de sus hogares, desarrollando actividades económicas desde ahí en otros emprendimientos económicos, ya sea individualmente o con sus parejas. Ello habla de la diversidad de prácticas de masculinidad que tienen lugar en el espacio doméstico, muchas de las cuales anteceden a las medidas de distanciamiento social. Las condiciones impuestas por el confinamiento son vivenciadas por estos varones como una variante más de las condiciones estructurales que moldean su experiencia de la masculinidad y los mandatos vinculados a ella (Olavarría, 2017), como la precarización del trabajo académico.

Las condiciones del mercado laboral académico han experimentado varios e importantes cambios en los últimos cincuenta años, lo que ha resultado en una experiencia muy

11 Cuya percepción económica está determinada por el número de horas de clase que se les asignan semestralmente. 
heterogénea de la labor académica con base en el momento histórico, administrativo e institucional en el que académicas y académicos se han incorporado a ella (Gil Antón, 2002). Esto se refleja en la vivencia de los varones entrevistados, entre los cuales hay jóvenes investigadores, profesores experimentados y varones que alternan su labor docente con otras actividades profesionales. Sin embargo, un elemento común en sus narrativas es la primacía que cobran su posición laboral y estatus contractual en relación con el mandato masculino de ser los principales proveedores materiales para ellos y sus familias, así como con las expectativas propias y de sus parejas sobre su responsabilidad en el mantenimiento del hogar y el cuidado de hijas e hijos (Olavarría, 2017).

Sergio (33), profesor de asignatura por más de diez años en la facultad donde realizó estudios de pregrado y posgrado, lo articula de la siguiente manera, al hablar de su decisión de rechazar una oferta para ser profesor investigador en una universidad en el norte del país:

(A)nalizándolo y platicándolo con mi esposa, me dijo: "si tú crees que [el salario] no es suficiente y que a la mejor nos vamos a tardar un rato en recuperarnos... pues, si quieres, nos quedamos aquí" [...] Desde luego, me dijo “tú decídelo” y al final decidí declinar la oferta [...] Sí por mí, pero también por mi familia, o sea, que estén tranquilos, que los hijos estén tranquilos. Porque, a lo mejor, yo diría "ya tengo mi plaza de investigación", que es lo que he estado buscando. Pero hay más gente junto a mí.

Años atrás, Sergio trabajó en la iniciativa privada por un tiempo, eso lo llevó a vivir fuera de la ciudad durante la semana y a viajar los fines de semana para volver a casa. Su experiencia en ese sentido fue difícil porque "extrañaba mucho la convivencia" con sus hijos. En aquel momento, ese acontecimiento influyó en su decisión de renunciar al empleo y regresar a dar clases a la facultad. Ahora, no estaba dispuesto a dejarles atrás para instalarse en otra ciudad, señala, pues considera que la cercanía física es fundamental para que sus hijos "estén tranquilos". Más aún, a pesar de haber concursado exitosamente por una plaza definitiva en otra universidad, Sergio valora que sus ingresos en esa posición no mejorarían sustancialmente la posición socioeconómica de su familia. Por ello, ha decidido apostar por la posibilidad, difícil según su apreciación, de incorporarse a la UNAM de manera similar.

Como Sergio, otros participantes en condiciones de trabajo menos estables relataron instancias en las que han buscado privilegiar la domesticidad por encima del avance en la 
carrera académica. Esta priorización aparece informada de manera muy importante por la materialidad de la vida y necesidades cotidianas, y su papel como proveedores materiales. Al mismo tiempo, como hace Sergio, otros varones articulan sus decisiones desde una dimensión simbólica, anidada en la crianza y la convivencia con hijas e hijos.

\section{Masculinidades y domesticidad: (con)vivencia en el confinamiento}

"En mi casa no me hallaba. Los fines de semana para mí eran como... bueno ya, el fin de semana y de vuelta al trabajo. Muchas vacaciones las he pasado metido en mi cubículo. Pero, curiosamente, esta situación no ha sido muy difícil para mí”, dice Octavio (66), investigador de tiempo completo desde hace varios años, al hablar sobre su vivencia del confinamiento. Ello pudiera estar relacionado, reflexiona, con el hecho de que pasa "buena parte del día pegado a la computadora, con el trabajo". Al mismo tiempo, le ha llevado a pensar acerca de su retiro, una situación que, debido a su edad, le parece cada vez más inminente y ha venido ponderando desde hace tiempo. Por ello, ha abordado el confinamiento como un "simulacro del retiro", aunque no del todo sin dificultad. Octavio vive con su esposa, quien por más de veinte años ha sido ama de casa. Eso le hace cuestionarse qué pasará cuando "el home-office", como él lo llama, no sea más un recurso para escapar de lo doméstico:

Hay momentos en que ella [su esposa] está haciendo el aseo de la casa y me bota para un lado y para otro. Yo le digo, bromeando, "por eso no me quiero retirar, porque tú, después de la bolsa de la basura, ¡me vas a sacar a mí!”. Y es verdad en parte, lo hemos platicado con amigos, que uno al retirarse cambia su vida drásticamente, y ellas siguen haciendo su vida como siempre. Lo hemos visto; las invadimos en nuestra propia casa, les estorbamos, para decirlo claro.

Como lo ilustra el relato de Octavio, los arreglos de género que construyeron lo doméstico en Occidente (y que, por tanto, se imbuyeron en los imaginarios y las prácticas de género en el México urbano de las clases medias y medias bajas) como un espacio, histórica y culturalmente, por y de mujeres han mostrado una notable resiliencia, a pesar de haber experimentado adecuaciones y contestaciones a lo largo del tiempo.

Así, el espacio doméstico es vivenciado por algunos participantes, especialmente aquellos en edades de 60 años en adelante, como un sitio donde la masculinidad tiene cabida, principal 
o exclusivamente, en algunas dimensiones específicas asociadas con un modelo convencional, como la de la proveeduría material o - de manera notable - la de la paternidad. Ignacio (69), profesor de carrera e investigador por más de cuarenta años, considera haber ejercido una masculinidad satisfactoria en lo doméstico en términos de proveeduría, pero también a través de su paternidad:

Asumía que yo tenía que trabajar y proveer dinero, porque llegamos a ese acuerdo al principio, ¿no?, yo trabajo y tú te encargas de los niños [...] pero, los reclamos son que yo era un padre ausente. Yo pienso que no era tan ausente, porque participaba en las fiestas infantiles, hasta haciendo... como mi trabajo es visual y creativo, hacía pasteles locos, y hacía piñatas locas y organizaba cosas locas. Pero cuando me reclaman es: "tú siempre estuviste ausente". Digo, bueno... no sé.

A pesar de que Ignacio considera haber estado presente en la crianza de sus hijas e hijos, y de que su exesposa realizaba también actividades profesionales mientras crecían, parece sentir que su actuación como padre es evaluada con un rigor excesivo. Según su relato, haber estado presente e involucrarse en momentos significativos (como las fiestas infantiles), realizando actividades de carácter lúdico y creativo en el espacio doméstico, es valorado por generaciones más jóvenes como insuficiente en términos de convivencia, cercanía e involucramiento paternos.

Los discursos sobre "nuevas masculinidades" enfatizan la incorporación de labores domésticas como la limpieza y el mantenimiento del hogar y, sobre todo, de cuidado de hijas e hijos (véase, por ejemplo, Boscan, 2008; Castillo y Morales, 2013), convencionalmente concebidas como femeninas, al repertorio de acciones masculinas legítimas y esperadas. Varones (auto)identificados como "nuevos" en la literatura tienden a asignar un valor importante a pasar tiempo con sus hijas e hijos, manifestar estar involucrados emocionalmente en su crecimiento y desarrollo, y declarar priorizar sus labores de paternidad por encima de otras actividades, cuando menos de manera discursiva. Asimismo, y a pesar de suscribir abiertamente discursos feministas y estar dispuestos a colaborar en el trabajo doméstico, también es posible observar en ellos una tendencia a priorizar el trabajo remunerado, el deporte o su estilo de vida, por encima de las labores domésticas (véase, por ejemplo, Gorman-Murray, 2008; Singleton y Maher, 2004). 
De manera notable, la concepción de Ignacio de su propia práctica masculina en conexión con lo doméstico no dista mucho de la prescrita por estos "nuevos" modelos de masculinidad. Al mismo tiempo, los relatos de la práctica doméstica de participantes, en edades entre 30 y 50 años, incluyen importantes rasgos de una masculinidad, designada como "nueva", que reproduce patrones convencionales de división sexual del trabajo y uso del tiempo. La vivencia de muchos de ellos puede ser capturada por la siguiente descripción de Gorman-Murray (2008):

Como compañero ideal de la "Nueva Mujer (trabajadora)", el Nuevo Hombre está íntimamente involucrado en la esfera doméstica, haciendo su parte del trabajo en casa — paternando, cocinando y limpiando. El Nuevo Hombre es, por tanto, un nuevo modelo de hetero-masculinidad moldeado a través de una (percibida) mayor participación en las actividades y el trabajo domésticos. Como resultado de su masculinidad doméstica, se espera que el hogar se desfeminice y el trabajo doméstico se des-generice (p. 371, traducción propia).

El contexto de confinamiento presenta una posibilidad particularmente propicia para varios participantes de interpretar y narrar su vivencia en términos de prácticas de "nueva masculinidad". Tales prácticas cumplen una importante función identitaria que permite a estos varones distanciarse de modelos "tradicionales", concebidos como negativos, y ubicarse en un proceso de reconfiguración de lo masculino, en particular en relación con lo doméstico.

Un ejemplo en este sentido es el de Arturo (36), profesor de carrera con importantes responsabilidades administrativas, quien relata haber disfrutado ampliamente estar en casa durante los periodos más álgidos de confinamiento. El trabajo de Arturo transcurre en más de una sede física de la universidad, lo que le implica pasar largos periodos fuera, y trasladándose desde y hasta su casa. El confinamiento le permitió experimentar el espacio doméstico de una manera particularmente idónea para el performance de esta nueva masculinidad:

(L)a casa pasó a tener multifunción, ¿no? Desde conectar a mis hijos a la escuela, que mi esposa tenía que hacer lo mismo por su lado, yo por mi lado. Fue aprender a respetar los horarios, porque cuando ella estaba en línea, yo 
conectaba a mis pequeños o les hacía la comida, bueno, los procuraba: los vestía, los bañaba. Pero cuando yo tenía que dar clase, pues ella también procuró muy bien que hasta guardaran silencio [...] [En el trabajo de investigación] no me pude enfocar, concentrarme y poder escribir; sentarme, aprovechar el tiempo para escribir o argumentar, no, la verdad no, no lo logré [risas]. Pero es algo que tampoco me causa preocupación o culpa, porque del otro lado, por ejemplo, a mis niños ahora ¡hasta les enseñé a jugar ajedrez! Entonces, unas por otras. Me gusta mucho avisar a los estudiantes: "quizá de un momento a otro viene alguno de mis hijos a saludarlos", incluir el espacio doméstico y que sepan que todos estamos en casa. Y sí, ocurrió un par de ocasiones, que llegaban, se asomaban y decían "hola", y todos los saludaban también de vuelta.

Dado que el confinamiento elimina la necesidad de dejar el hogar físicamente para realizar el trabajo académico, el confinamiento otorga posibilidades a los varones para involucrarse en la labor doméstica, e incorporar la domesticidad, aunque sea de maneras tangenciales, al trabajo académico, como lo señala Arturo. No obstante, se infiere que se trata de algo transitorio, que tendería a retomar su cauce una vez superada la contingencia y reanudada la "normalidad" del espacio público (Arturo señala que ha retomado algunas labores de manera presencial). Esto sugiere, por un lado, la necesidad de una potencial negociación entre la imagen auto adscrita de varones comprometidos e involucrados en la labor doméstica, y por otro, las limitaciones materiales que puedan imponerse a la conclusión del confinamiento, cuando estos varones vuelvan a pasar una gran parte de su tiempo en el espacio profesional, y fuera del hogar familiar.

Las actividades de crianza o cuidado, descritas en varios casos como posibles fuentes de gratificación son producidas en varios relatos como estructuralmente más restringidas para los varones que trabajan fuera del hogar habitualmente. El trabajo académico profesional aparece en algunas de las narraciones como estructural e institucionalmente vinculado al espacio público. Aunado a su estatus como fuente primaria de proveeduría material, el trabajo académico es vivenciado como una labor masculinizada en un sentido "tradicional". Ello, entorpecería el desarrollo de modelos de "nueva masculinidad" como el descrito por Gorman-Murray. 
El relato de Ernesto, técnico académico y docente de 63 años, es interesante en ese sentido. Mientras estuvo casado, participó activamente en la crianza y cuidado de su hijo, puesto que su trabajo en esos momentos así se lo permitía. Hasta antes de la contingencia sanitaria pasaba grandes periodos en su escuela (fuera del campus central de la universidad) con horarios poco flexibles. Las circunstancias excepcionales del confinamiento, considera Ernesto, establecieron las condiciones para ser cuidador de nueva cuenta, esta vez de su madre:

Un día llegué [a casa de su madre] y estaba muy mala. Le dije "te vas conmigo" y me la traje a mi departamento y la atendí. Recuerdo que una noche empezó a subirle muy rápido la temperatura... traté de bajársela como pude, no lo logré, y ya cuando tenía 39.5, la llevé a una clínica aquí al lado, prácticamente cargando porque apenas podía caminar, y ahí me dijeron “tiene todos los síntomas [de COVID], eh”. Le hicimos la prueba y salió positiva [...] y estuve cuidándola: dándole los medicamentos, la dieta, los cuidados; dormir, descansar, muchísima agua, pararla para que no estuviera acostada todo el tiempo. Y sobrevivió. Y de no haber sido por esta situación no hubiera tenido el privilegio de atender a mi mamá y prácticamente salvarla de la muerte [...] Ahora ya regresé a la escuela, a otro ritmo, pues, pero ya yendo. Y bueno, la visito [a su madre], así que por ese lado agradezco a la vida haber tenido esa oportunidad de cuidar a mi madre y de conocerla de esa manera.

Ernesto otorga un gran significado a su labor de cuidado en esta experiencia mediante la cual su espacio doméstico se reconfiguró como uno de cuidado de su madre, su principal cuidadora en su etapa de crecimiento. Según su apreciación, esta experiencia difícilmente hubiera sido posible en condiciones "normales", puesto que estaría condicionada a la posibilidad de trabajar remotamente y demás circunstancias impuestas por el confinamiento.

Por otro lado, en el caso de otros participantes, la actividad académica es experimentada como más favorable para la actuación y el desempeño de modelos de masculinidad que incorporan el cuidado y la crianza. Especialmente, este es el caso de quienes habitualmente desempeñan sus labores dentro de los límites del campus central de la universidad, poseen nombramientos académicos permanentes y en general gozan de mayor estabilidad laboral: 
(E)n la universidad como que uno puede acomodar más sus tiempos, de hecho, una de las cosas que me hizo ver que quería tener una vida académica fue eso, que dije "si voy a tener una familia, si voy a tener hijos, pues sí me gustaría”. Como que combina ¿no? Y [su esposa], desafortunadamente, no tiene esa holgura, o esa posibilidad (Jorge, 40, técnico académico).

La verdad es que la universidad ha sido muy noble, [su hijo] ha crecido en la universidad. El camión [escolar] lo deja ahí, en rectoría. Vamos [su esposa y él] por él, lo tenemos ahí en el laboratorio haciendo tareas; ha crecido mucho ahí, en los espacios de la universidad. (Manuel, 46, profesor de carrera e investigador).

Desde antes de separarnos [su exesposa y él], yo me hacía cargo de [su hija], iba por ella a la guardería, al kínder, etcétera y me la llevaba al trabajo. Y la cuidaba ahí en el centro de cómputo; hay fotos mías cambiándole el pañal y cosas de estas, ¿no? Ya después, en la noche íbamos por la mamá, íbamos todos a la casa. Pero siempre me hice cargo de ella. Y, como quiera, es asumir el papel de papá soltero, que no era siempre bien visto, o bien entendido; como que no es común, entonces mucha gente no lo comprende (Adrián, 58, profesor de carrera e investigador).

Los testimonios anteriores ilustran la posibilidad de algunos varones de apropiarse del espacio laboral universitario e impregnarle un carácter doméstico, enfatizando la fluidez que dichos espacios pueden llegar a tener. Al mismo tiempo, sus relatos apuntan hacia la multiplicidad de la experiencia académica, derivada de la organización de la masculinidad en el ámbito académico. Así, la masculinidad académica manifiesta una dimensión "convencional" en términos de las expectativas de la práctica de los varones, como lo sugiere lo relatado por Adrián, en el sentido de que la figura de padre soltero puede resultar ininteligible para algunas personas, y la manera en que la disposición espacial del trabajo académico desvincula a los varones de ciertas labores domésticas que, para algunos de ellos, además de necesarias, resultan gratificantes. 
Por otro lado, la experiencia académica (masculina) también exhibe una dimensión jerárquica, si bien de manera diferenciada por áreas de conocimiento y/o administrativas al interior de la universidad. Adrián, por ejemplo, realiza sus labores académicas en el área de las humanidades. Por su parte, Gustavo (46), técnico académico y docente en el área de conocimiento de las llamadas "ciencias duras", considera que la flexibilidad en el trabajo para hacer frente a las labores domésticas y de cuidado es menor en su contexto, en el que se espera que la prioridad sea su actividad profesional. Ello se agudiza, señala, si se tienen responsabilidades administrativas (de coordinación, jefaturas, etcétera). En su experiencia, a medida que el confinamiento se ha ido relajando y las actividades (en línea o presenciales como en el caso de Ernesto) se van incrementando, el trabajo académico comienza a absorber al varón "de manera inercial", y empieza a dificultar su participación en las labores domésticas, aun si se trabaja desde casa.

\section{Aislamiento social en tiempos de pandemia: masculinidades académicas "frente al espejo"}

"Estar con otra persona te confronta muchas cosas que tú ya sabías evadir", es parte de la reflexión de Raúl (46) respecto a cómo ha sido convivir las 24 horas de cada día con su esposa, por varios meses seguidos. "(C)uando vives solo", —elabora_ "no te das cuenta de muchas cosas de ti mismo, porque ya estás tan acostumbrado a tu persona que ya sabes cómo darte la vuelta, engañarte y decirte que todo está bien, cuando pues no necesariamente todo está bien todo el tiempo".

El trabajo académico, especialmente de investigación como el que realiza Raúl, fue descrito por varios de los participantes como solitario en muchos sentidos: más de uno evocó en sus narraciones la imagen del profesor investigador sentado frente a la computadora, por horas, en la soledad de su cubículo. Ese aislamiento ocurre en el marco de lo que podemos caracterizar, siguiendo a Connell, como el "régimen de género" (Connell y Pearse, 2015) de la universidad; esto es, una serie de arreglos relacionales y prácticas de género que ordenan la convivencia en su interior y con relación a ella.

En este sentido, es posible pensar en masculinidades académicas que se producen y reproducen en la intersección de una masculinidad hegemónica — configurada con arreglo 
al orden de género- - y los arreglos relacionales y las prácticas de género específicas que dan forma a la experiencia de los varones académicos que laboran en la UNAM. Así, las masculinidades académicas desplegadas por los participantes pueden ser entendidas como expresiones de la hegemonía en el contexto universitario específicamente, que se materializan, por ejemplo, en una amplia gama de posibilidades para la experiencia masculina en solitario, entre ellas la evasión a la que refiere Raúl.

De manera paradójica, el aislamiento social impuesto por "los tiempos de pandemia", como los llama Raúl, irrumpió en los espacios y momentos solitarios a los que los académicos suelen tener acceso, y los colocó en una posición en la que evadir una serie de relaciones, prácticas y significados sobre lo masculino y lo femenino, que tienen lugar en el espacio doméstico, es prácticamente imposible. Como lo expresa Mario (41), investigador de tiempo completo, pasar todo el tiempo en presencia de otras personas de su familia "es como vivir en una casa llena de espejos por todos lados". Ello reduce los espacios donde la masculinidad no se vea reflejada y donde sus aspectos problemáticos puedan ser invisibilizados e ignorados, como en los confines de los institutos y centros de investigación.

Dicha circunstancia es vivida de manera diferenciada por los académicos participantes en este estudio. Notablemente, aquellos pertenecientes a las generaciones más jóvenes hablaron de manera más amplia sobre su condición como varones académicos frente a sus parejas, hijas e hijos, y otras personas con quienes tienen relación. Su vivencia del confinamiento aparece informada por una cierta reflexividad que, señalan, había iniciado previamente. Esto se asemeja a lo descrito por Córdoba e Ibarra (2020) en un estudio con varones durante el confinamiento en Chile; los autores señalan que algunos de sus entrevistados se encontraban ya inmersos en un "proceso de deconstrucción masculina" (p. 63) cuando inició el confinamiento, lo que les ha ayudado a sortearlo mejor. Para otros, consignan, esta circunstancia excepcional ha representado la oportunidad de iniciar ese proceso, "potenciando su capacidad empática en sus vínculos afectivos, con hijos, hijas, parejas y otros allegados, y generando corresponsabilidad doméstica y parental” (p. 64).

Al hablar respecto a la realización y distribución de tareas de trabajo doméstico y las expectativas en ese sentido (tanto propias, como de su pareja, de otras personas en su familia, o de "lo esperado socialmente"), algunos varones que cohabitan con sus parejas recurrieron a frases como "aquí nos repartimos todo", "lo hacemos entre ambos", "tratamos de 
distribuirlo de manera equitativa", o "lo vamos haciendo conforme vamos pudiendo". Tales expresiones remiten a un esfuerzo constante por desplegar una convicción sobre la importancia de distribuir las labores domésticas de forma equitativa. Dicho esfuerzo aparece informado por discursos igualitarios, ampliamente presentes en los espacios universitarios, que estos varones incorporan a sus narrativas en diferentes niveles.

Arturo lo describe mediante un ejercicio comparativo entre su vivencia de la domesticidad en su familia de origen y su familia actual:

Antes cuestionaba un poco más [algunos posicionamientos feministas], pero ahora he buscado adoptar más el escuchar. Su perspectiva, su posición. No solamente de la violencia que viven [las mujeres], que no es menor, sino pues de otros aspectos políticos, etcétera y eso pues no lo he aprendido desde niño. Porque yo sé que mi familia nuclear ha sido muy patriarcal. No sé si tanto como machista, pero sí ha sido una familia muy de roles tradicionales [...] Y sí, sin lugar a duda, la educación de mis hijos, de mis hijas, que procuro, o procuramos, mi esposa y yo, tratarlos parejo en todo.

Arturo señala que estos planteamientos igualitarios no estaban presentes en la domesticidad en la que creció. Su socialización reafirmó en él la noción de que su condición como varón lo habilitaba mejor para la interacción en el espacio público (él y sus hermanos tenían más autonomía que sus hermanas para salir de casa y permanecer fuera por periodos prolongados) y configuró su masculinidad en estrecha relación con el espacio extradoméstico. La percepción de Arturo de estar enmarcado por un régimen de género universitario que incorpora crecientemente discursos igualitarios interactúa con su convivencia con personas importantes y significativas para él (como su esposa o algunas de sus colegas y alumnas) y, notablemente, con la experiencia de la paternidad. Ello resulta en el desarrollo de una mayor sensibilidad respecto a las desigualdades de género en la cotidianidad y en la configuración de los espacios universitarios.

La mayoría de los participantes en el estudio han tenido contacto cercano con iniciativas para la sensibilización en género, la difusión de las problemáticas en ese sentido al interior de la universidad, y la transversalización institucional de la perspectiva de género. Esto coincide con lo planteado por Daniela Cerva (2018) en su estudio sobre la politización del 
género entre varones universitarios, en el sentido de que las posiciones más reflexivas son producidas precisamente por aquellos académicos que han estado involucrados en proyectos, talleres u otras actividades con temáticas y enfoques de género, organizadas por instancias universitarias o desarrolladas al interior de la institución. Esto sugiere que la movilización de las mujeres para la denuncia y el combate a la problemática de género, así como algunas de las iniciativas institucionales abocadas a dichas tareas, han tenido ciertos efectos positivos, aunque limitados. Al mismo tiempo, apunta a la necesidad de profundizar sus objetivos, alcance y ámbitos de acción.

No obstante, en la mayoría de los casos existe una admisión, a veces explícita pero generalmente implícita, de que la balanza del trabajo doméstico tiende a inclinarse hacia sus parejas femeninas. En muchos casos, son ellas quienes "coordinan" las labores domésticas, desde la asignación de tareas o la elección de comidas, hasta la gestión de la relación contractual con las trabajadoras del hogar a quienes emplean. El caso de Octavio, al que me referí en la sección anterior, es un ejemplo claro de que la construcción de lo doméstico como mayor o exclusivamente femenino tiene aún una gran relevancia simbólica. El confinamiento parece haber visibilizado dicha circunstancia, pero esto no ha significado, necesariamente, que todos los varones se hayan involucrado en un proceso de reflexión al respecto.

\section{Conclusiones}

El confinamiento, como referente material y simbólico de "la pandemia", enfatiza de maneras variadas los procesos mediante los cuales las prácticas e identidades masculinas, de los académicos entrevistados, están influidas por una serie de condiciones estructurales que configuran el orden de género y vinculan lo masculino con el espacio público. Asimismo, los relatos de estos varones sobre su presencia y actuación en el hogar impuesta por el confinamiento arrojan luz sobre la dimensión doméstica de su práctica masculina, y permiten repensar la masculinidad, y observar matices en ella desde lo doméstico, al ser habitado por estos varones por periodos inusualmente prolongados y de maneras múltiples. Así, el espacio doméstico obtiene, por momentos, un cariz más masculino del habitual.

En sus narrativas sobre el confinamiento, los participantes presentan grados diferenciados de reflexividad respecto a las desigualdades derivadas por la configuración género-espacial 
y los vínculos que establece entre masculinidad y espacio público (y, por extensión, feminidad y espacio privado), y los privilegios derivados de ella. No obstante, en las narrativas de todos los participantes, dicha configuración aparece aún como un elemento constitutivo y delimitante de su práctica masculina.

Resulta especialmente notable la naturalización del trabajo doméstico remunerado como una actividad feminizada sobre la cual descansa, de manera importante, la labor académica (sea ésta realizada por varones o por mujeres). El confinamiento representa una crisis transitoria de ese modelo de distribución generizada de las tareas domésticas que obliga a los varones a adaptarse y realizar varias de esas labores, en algunos casos por primera vez en sus vidas, mientras se supera la crisis.

Ello ha favorecido que algunos de estos varones reflexionen sobre la validez y pertinencia de la configuración género-espacial a la que me he referido, aunque esto no se verifique necesariamente en todos los casos. Asimismo, ha evidenciado la posibilidad de reconfiguraciones en las prácticas de género en el espacio doméstico, sin que esto haya implicado cambios sustanciales en las relaciones e identidades de género al interior de los hogares. Las adecuaciones a las dinámicas de género en lo doméstico parecen obedecer más a las condiciones materiales impuestas por la coyuntura que a un proceso reflexivo profundo y significativo derivado de ella. Será importante dar seguimiento a esas reflexiones y reconfiguraciones generadas en algunos de estos casos a medida que el confinamiento y el distanciamiento social se van relajando, y las actividades en el espacio público comienzan a regularizarse.

El hecho de que las condiciones impuestas por el confinamiento generen en los participantes reflexiones respecto a los privilegios a los que pueden acceder como varones a través del trabajo académico es también notable. En este sentido, son destacables sus observaciones en torno a cómo dichos privilegios se distribuyen de manera diferenciada, en función de factores percibidos con base en el mérito (su antigüedad en la actividad académica, el grado académico con el que cuentan), pero también como parte de una estructura jerarquizada por elementos que sugieren la existencia y operación de esquemas de masculinidad hegemónica (Messerschmidt, 2018). Tales observaciones apuntan a una resignificación del trabajo académico no sólo como fuente de provisión material y realización profesional, sino como 
un elemento central de la práctica de género en el espacio doméstico, y revelan posibilidades para reconfigurar sus prácticas domésticas.

Si bien la mayoría de los entrevistados manifestaron abiertamente su disposición y necesidad de participar en actividades domésticas, éstas aparecen en sus relatos como estructuralmente más restringidas para los varones y más adecuadas en ese sentido a la labor y experiencia femeninas. Sus razonamientos están enmarcados por la vinculación estructural y cultural del trabajo académico con el espacio público, por su estatus como fuente primaria de proveeduría material, y por su carácter jerarquizado. De forma interesante, dicha situación aparece en las narrativas de los participantes mayormente como no problemática; es la fase crítica del confinamiento la que impone dificultades al desarrollo armónico de la división sexual del trabajo. Una notable excepción en este sentido es la relacionada con las actividades de crianza o cuidado, descritas en algunos casos como potenciales fuentes de gratificación, sobre todo en su dimensión más lúdica.

\section{Agradecimientos}

Agradezco a las integrantes del Seminario Permanente de Investigación de Género del CIEGUNAM por sus valiosos comentarios y observaciones a este artículo. Un agradecimiento especial a Nallely García por su apoyo en la administración y procesamiento de los datos.

\section{Referencias bibliográficas}

Amuchástegui, Ana y Szasz, Ivonne. (2007). El pensamiento sobre masculinidades y la diversidad de experiencias de ser hombre en México. En Ana Amuchástegui e Ivonne Szasz (Coords.), Sucede que me canso de ser hombre. Relatos y reflexiones sobre hombres y masculinidades (pp. 15-38). México: El Colegio de México.

Andrews, Molly; Day Sclater, Shelley; Squire, Corinne y Tamboukou, Maria. (2004). Narrative research. En Clive Seale, Giampietro Gobo, Jaber Gubrium y David Silverman (Eds.), Qualitative Research Practice (pp. 97-112). London: SAGE.

Boscán, Antonio. (2008). Las nuevas masculinidades positivas. Utopía y Praxis Latinoamericana. $\quad$ 13(41), 93-106. Recuperado de https://produccioncientificaluz.org/index.php/utopia/article/view/2811 
Buquet, Ana. (2016). El orden de género en la educación superior: una aproximación interdisciplinaria. Nómadas, 44, 27-43. Recuperado de http://editorial.ucentral.edu.co/ojs_uc/index.php/nomadas/article/view/2487

Buquet, Ana; Cooper, Jennifer; Mingo, Araceli y Moreno, Hortensia. (2013). Intrusas en la universidad. México: Universidad Nacional Autónoma de México, PUEG, IISUE.

Byrne, Bridget. (2016). Qualitative Interviewing. En Clive Seale (Ed.), Researching Society and Culture (pp. 217-236). Londres: SAGE.

Carrasquer, Pilar; Torns, Teresa; Tejero, Elisabet y Romero, Alfonso. (1998). El trabajo reproductivo. Papers. Revista de Sociología, 55, 95-114. doi: http://dx.doi.org/10.5565/rev/papers. 1934

Castellanos-Torres, Esther; Mateos, José y Chilet-Rosell, Elisa. (2020). COVID-19 en clave de género. Gaceta Sanitaria, 34(5), 419-21. doi: 10.1016/j.gaceta.2020.04.007

Castillo, Jennifer y Morales, Helena. (2013). Los estudios de género a las nuevas masculinidades y/o los movimientos de padres por la custodia compartida de sus hijos e hijas, Educación y Humanismo, 15(24), 107-121. Recuperado de http://revistas.unisimon.edu.co/index.php/educacion/article/view/2208

Cerva, Daniela. (2018). Masculinidades y educación superior: la politización del género. $E l$ Cotidiano. Revista de la realidad mexicana, 212, 35-45.

Connell, Raewyn. (1983). Men's bodies. En Raewyn Connell, Which way is up? Essays on class, Sex and Culture (pp. 17-32). Sidney: George Allen \& Unwin.

Connell, Raewyn. (1987). Gender and Power: Society, the Person, and Sexual Politics. Chichester: Wiley

Connell, Raewyn. (2015). Masculinidades. México: Universidad Nacional Autónoma de México, PUEG. 
Connell, Raewyn y Pearse, Rebecca. (2015). Gender: in world perspective. Cambridge: Polity Press.

Córdoba, María e Ibarra, Darío. (2020). ¿Varones construyendo espacios de Igualdad? Desafíos en contexto de confinamiento (COvID-19). Revista Punto Género, (13), 5065. doi: https://doi.org/10.5354/0719-0417.2020.58191

Cruz, Adriana; Noriega, Mariano y Garduño, María. (2003). Trabajo remunerado, trabajo doméstico y salud: las diferencias cualitativas y cuantitativas entre mujeres y varones. Cadernos de Saúde Pública, 19, 1129-1138.

Departamento Administrativo Nacional de Estadística, DANE. (2020). Tiempo de cuidados: las cifras de la desigualdad. Bogotá: Departamento Administrativo Nacional de Estadística (DANE), ONU Mujeres.

del Río, María y García, María. (2020). Cuidados y abordaje de la pandemia de COVID-19 con enfoque de género. Gaceta Sanitaria. doi: https://doi.org/10.1016/j.gaceta.2020.05.006

Eberle, Thomas. (2014). Phenomenology as a Research Method. En Uwe Flick (Ed.), The SAGE Handbook of Qualitative Data Analysis (pp. 184-202). Londres: SAGE.

Esin, Cigdem; Fathi, Mastoureh y Squire, Corinne. (2014). Narrative Analysis: The Constructionist Approach. En Uwe Flick (Ed.), The SAGE Handbook of Qualitative Data Analysis (pp. 203-216). Londres: SAGE.

Espino, Alma. (2010). Economía feminista: enfoques y propuestas. Montevideo: Universidad de la República, Instituto de Economía, Serie Documentos de Trabajo $5 / 10$.

Figueroa, Juan G. (Coord.) (2014). Políticas públicas y la experiencia de ser hombre: paternidad, espacios laborales, salud y educación. México: El Colegio de México, CEDDUA 
Gil Antón, Manuel. (2002). Amor de ciudad grande. Una visión general del espacio para el trabajo académico en México. Sociológica, 17(49), 93-130.

Gorman-Murray, Andrew. (2008). Masculinity and the Home: A Critical Review and Conceptual Framework. Australian Geographer, 39(3), 367-379.

Gorman-Murray, Andrew. (2013). Urban Homebodies: Embodiment, Masculinity, and Domesticity in Inner Sydney. Geographical Research, 51(2), 137-44.

Instituto Nacional de Estadística y Geografía, INEGI. (2019). Encuesta Nacional sobre el Uso del Tiempo (ENUT) 2019: presentación de resultados. Aguascalientes: Instituto Nacional de Estadística y Geografía, INEGI.

Instituto de las Mujeres de la Ciudad de México. (2017). Gaceta 3: Trabajo doméstico y de cuidado no remunerado. México: Instituto de las Mujeres de la Ciudad de México.

Lohokare, Madhura (2020). Locating critical masculinities theory. Masculinities and space and place. En Lucas Goettzen, Ulf Mellström y Tamara Shefer (Eds.), Routledge International Handbook of Masculinity Studies (pp. 292-301). Nueva York: Routledge.

López-Hernández, Edenia y Rubio-Amores, Dominique. (2020). Reflexiones sobre la violencia intrafamiliar y violencia de género durante emergencia por COVID-19. CienciAmérica, 9(2), 312-321. doi: http://dx.doi.org/10.33210/ca.v9i2.319

Martínez-Restrepo, Susana; Tafur, Lina; Osio, Juan y Cortés, Pablo. (2020). Violencias basadas en género en tiempos de Covid-19. Centro de Investigación Económica y Social, $\quad$ Fedesarrollo. $\quad$ Recuperado de https://www.repository.fedesarrollo.org.co/handle/11445/4012

Mason, Jennifer. (2002). Qualitative researching. Londres: SAGE.

Messerschmidt, James. (2018). Hegemonic Masculinity. Formulation, reformulation and amplification. Lanham: Rowman and Littlefield. 
Olavarría, José. (2001). ¿Hombres a la deriva? Poder, trabajo y sexo. Santiago: Facultad Latinoamericana de Ciencias Sociales, FLACSO.

Olavarría, José. (2017). Sobre hombres y masculinidades: ponerse los pantalones. Santiago: Universidad Academia de Humanismo Cristiano, UAHC.

Pedrero, Mercedes. (2004). Género, trabajo doméstico y extradoméstico en México. Una estimación del valor económico del trabajo doméstico. Estudios Demográficos y Urbanos, 19(2), 413-446. doi: http://dx.doi.org/10.24201/edu.v19i2.1191

Ríos, Nicolás; Mandiola, Marcela y Varas, Alejandro. (2017). Haciendo género, haciendo academia: un análisis feminista de la organización del trabajo académico en Chile. $\begin{array}{llll}\text { Psicoperspectivas. Individuo } \quad y \quad \text { Sociedad, } & \text { 16(2), }\end{array}$ doi: https://dx.doi.org/10.5027/psicoperspectivas-vol16-issue2-fulltext-1041

Rojas, Georgina y Contreras, Nidia. (2018). Resistencia activa de las trabajadoras del hogar en México: talleres, aprendizaje y empoderamiento. Revista Latinoamericana de Antropología del Trabajo, 2(3). Recuperado de http://www.ceilconicet.gov.ar/ojs/index.php/lat/article/view/346.

Ruiz, María. (2020). Las estadísticas sanitarias y la invisibilidad por sexo y de género durante la epidemia de COVID-19. Gaceta Sanitaria. 35(1), 95-98. doi: https://doi.org/10.1016/j.gaceta.2020.04.008

Ruiz-Pérez, Isabel y Pastor-Moreno, Guadalupe. (2020). Medidas de contención de la violencia de género durante la pandemia de COVID-19. Gaceta Sanitaria. doi: https://doi.org/10.1016/j.gaceta.2020.04.005

Singleton, Andrew y Maher, Jane Maree. (2004). The "New Man" is in the House: Young Men, Social Change, and Housework. The Journal of Men's Studies, 12(3), 227-240. Doi: https://doi.org/10.3149/jms.1203.227 
Schutz, Alfred. (1967 [1932]). The Phenomenology of the Social World (Trads. George Walsh y Frederick Lehnert). Evanston, IL: Northwestern University Press.

Smith, Glendon y Winchester, Hilary. (1998). Negotiating space: alternative masculinities at the work/home boundary. The Australian Geographer, 29(3), 327-339. doi: https://doi.org/10.1080/00049189808703227

Wenham, Clare; Smith, Julia y Morgan, Rosemary. (2020). COVID-19: the gendered impacts of the outbreak. The Lancet, 395(10227), 846-848. doi: https://doi.org/10.1016/S01406736(20)30526-2

\section{ALÍ SILES}

Es doctor en Sociología por la Universidad de Manchester, Reino Unido. Investigador Asociado en el Centro de Investigaciones y Estudios de Género (CIEG) de la Universidad Nacional Autónoma de México. Miembro de la Latin American Interdisciplinary Gender Network (LAIGN), auspiciada por la Universidad de Yale, el CIEG y la UNAM-Boston. Sus principales líneas de investigación son: hombres y masculinidad(es), género, vida personal y cotidiana, identidad(es) y subjetividad(es). 